\title{
The prevalence of microRNA binding sites around the CGA, $F S H B, L H B$ and TSHB genes in mammals with a different number of dominant follicles
}

\author{
O.V. Lyangasova, M.A. Amelina, T.P. Shkurat* \\ Southern Federal University, Rostov-on-Don, Russia \\ *e-mail: tshkurat@yandex.ru
}

Key words: microrna, $C G A, F S H B, L H B, T S H B$, dominant follicle

Motivation and Aim: Folliculogenesis is a constant process of the hierarchy of follicles, in which the growth and maturation of some follicles and atresia of others occurs simultaneously. Both the number of simultaneously maturing dominant follicles and the number of young are an evolutionarily fixed feature of the species. The genes that regulate the time and number of ripening follicles are practically the same in all mammals. The system of regulation of these genes can impact on the onset of ovulation in ontogeny and on the number of simultaneously maturing follicles. The aim of the study was to perform a comparative bioinformation analysis of the localization of microRNA binding sites in introns and intergenic space of $C G A$ (gonadotrophic hormone alpha chain), $F S H B$ (follicle stimulating hormone beta chain), $L H B$ (luteinizing hormone beta chain) and TSHB (thyroid-stimulating hormone beta chain) genes in mammals with one and more simultaneously maturing dominant follicles.

Methods and Algorithms: The genomes of animals with a different number of dominant follicles have been used. The animals with only one dominant follicle: Homo sapiens, Ovis aries, Bos taurus, Gorilla gorilla, Macaca mulatta, Pan troglodytes, Pongo abelii, Carlito syrichta, Equus caballus, Vicugna pacos, Otolemur garnettii, Loxodonta africana, Nomascus leucogenys, Myotis lucifugus. The animals with several dominant follicles: Canis lupus, Musmus culus, Oryctolagus cuniculus, Sus scrofa, Rattus norvegicus, Cavia porcellus, Mustela putorius furo, Felis catus, Tupaia belangeri, Echinopstel fairi, Sorex araneus, Erinaceus europaeus, Ochotona princeps. The microRNA sequences for homologous search were taken from the miRBase database (http:// mirbase.org/). The bioinformatic analysis was carried out with the Mscanner software developed by us (Shkurat et al., 2015).

Results: A high correlation of the number of microRNA binding sites with some studied indicators of the reproductive system was found (the age of maturation in females, the number of broods per year, the duration of ovulation, the length of pregnancy, the time interval between births, the weight of the birth-weight, the weight of the female). The number of microRNA binding sites around the $C G A$ gene in all animals positively correlated with the duration of pregnancy $\mathrm{r}=0.89$; the birth weight $r=0.86$; the interval between genera $r=0.79$ and with the lifetime $r=0.7$. A negative correlation was found with the duration of ovulation (estrus) $r=-0.83$; the number of young in the brood $\mathrm{r}=-0.82$; the number of broods per year $\mathrm{r}=-0.74$. The number of microRNA binding sites around the $F S H B$ gene positively correlated with the age at onset of puberty in females $r=0.77$, the interval between genera $r=0.87$, the number of broods per year $r=0.74$, the lifespan $\mathrm{r}=0.79$, the cycle duration $\mathrm{r}=0.68$. More than 4 million pairs of nucleotides were analyzed, 5,967 coincidences with the mature microRNA sequences recorded in the miRBase database were found. 167 to 262 mature microRNA sequences are located in the cis-regulatory regions of the $C G A$ gene in animals with the only one dominant follicle, which is almost twice as large compared to the group of animals with several simultaneously maturing follicles $(n=68-130)$. 267 to 367 sequences of mature microRNAs are located in the FSHB gene in animals with the only one dominant follicle, which significantly exceeds these values in the group of animals with several dominant follicles $(\mathrm{n}=99-217)$. There are 1 to 9 microRNA binding sites around the $L H B$ gene in all animals, and 11 to 219 around the TSHB gene, without correlating with the number of dominant follicles.

Acknowledgements: The study was performed in the framework of the basic part of the Ministry of education and science of the Russian Federation state task, project No. 6.6762.2017/BT. 